\title{
Consomic Mouse Strain
}

National Cancer Institute

\section{Source}

National Cancer Institute. Consomic Mouse Strain. NCI Thesaurus. Code C14373.

Strains of mice derived by repeated backcrossing while maintaining one whole chromosome genetically different from the background strain. (IAX IMSR glossary) 